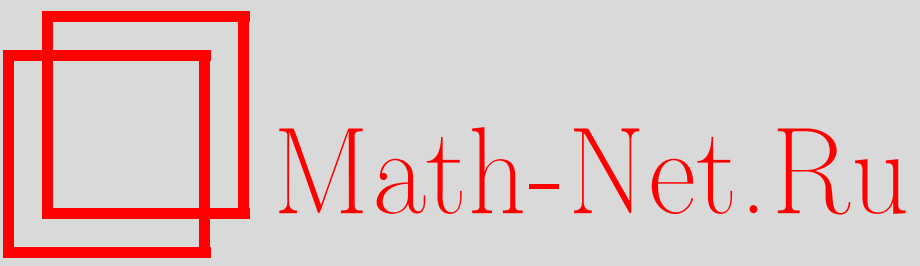

Р. Эрнандес Эредеро, Интегрируемые квазилинейные уравнения, ТМФ, 2002, том 133, номер 2, 233-246

DOI: https://doi.org/10.4213/tmf393

Использование Общероссийского математического портала Math-Net.Ru подразумевает, что вы прочитали и согласны с пользовательским соглашением

http://www.mathnet.ru/rus/agreement

Параметры загрузки:

IP : 18.234 .197 .8

26 апреля 2023 г., 16:58:31 
ТЕОРЕТИЧЕСКАЯ

И МАТЕМАТИЧЕСКАЯ

ФИЗИКА

Том 133, № 2

ноябрь, 2002

(C) 2002 r.

\section{ИНТЕГРИРУЕМЫЕ КВАЗИЛИНЕЙНЫЕ УРАВНЕНИЯ}

Развита схема, позволяющая классифицировать интегрируемые скалярные эволюционные уравнения третьего порядка с использованием симметрийного подхода к интегрируемости. Эта схема применена к исследованию квазилинейных уравнений специального вида, что позволило доказать, что некоторые из этих уравнений, которые, как ожидалось, должны быть интегрируемы, могут быть сведены к хорошо известным уравнениям Кортевега-де Фриза и Кричевера-Новикова с помошью дифференциальных подстановок типа Миуры.

Ключевые слова: классификация интегрируемых дифференциальных уравнений, формальный симметрийный подход, дифференциальные подстановки.

\section{1. ВВЕДЕНИЕ}

Проблема идентифицирования и классификации интегрируемых уравнений вида

$$
w_{t}=F\left(t, x, w, w_{x}, w_{x x}, w_{x x x}\right)
$$

с использованием так называемого симметрийного подхода представляет собой классическую задачу, возникающую при исследовании свойств интегрируемости. В рамках этой задачи было получено множество частных и разрозненных результатов [1]-[6]. Вычислительная сложность задачи приводит к тому, что до сих пор имеются многочисленные пробелы в классификации и многие предположения остаются недоказанными. Тем не менее современная компьютерная техника и разработанные пакеты программ позволяют выйти на такой уровень вычислений, который дает возможность заполнить указанные пробелы.

Главное предположение в предыдуших исследованиях состояло в том, что все интегрируемые уравнения из семейства (1) могут быть приведены либо с помошью классического (точечного или контактного) преобразования, либо в результате более сложной дифференциальной подстановки (типа преобразования Миуры или преобразования Коля-Хопфа [7], см. ниже (23)) к одному из трех фундаментальных интегрируемых уравнений:

*Departamento de Física Teórica II, Facultad de Ciencias Físicas, Universidad Complutense de Madrid, Madrid, Spain. E-mail: rafahh@fis.ucm.es 


$$
\begin{aligned}
& w_{t}=w_{x x x}+\alpha(x) w_{x}+\beta(x) w \\
& w_{t}=w_{x x x}+w w_{x} \\
& w_{t}=w_{x x x}-\frac{3}{2} w_{x}^{-1} w_{x x}^{2}+\left(4 w^{3}+g_{1} w+g_{2}\right) w_{x}^{-1}+c w_{x}
\end{aligned}
$$

т.е. или к линейному уравнению, или к уравнению Кортевега-де Фриза (КдФ), или к уравнению Кричевера-Новикова $(\mathrm{KH})$.

Общее утверждение симметрийного подхода к интегрируемости [5], [8]-[10] состоит в том, что любое интегрируемое уравнение допускает последовательность так называемых канонических локальных законов сохранения

$$
\left(\rho_{i}\right)_{t}=\left(\sigma_{i}\right)_{x}, \quad i=1,2, \ldots
$$

Канонические плотности $\rho_{i}$ могут быть явно записаны в терминах величин в правой части уравнения (1) и в терминах потоков $\sigma_{j}, j=1, \ldots, i-1$ (механизм получения таких формул подробно описан в работе [4]). Система уравнений (5) при этом преврашается в набор условий интегрируемости для семейства уравнений (1). Неизвестная, которую надо найти, - это функция $F$, которая, будучи найдена, порождает полный набор интегрируемых уравнений из семейства (1). Система уравнений (5) столь переопределена, что уже наложение нескольких первых условий интегрируемости обычно оказывается достаточным для нахождения всех интегрируемых случаев.

В данной работе получены следующие результаты. Во-первых, получила дальнейшее развитие предварительная классификация из работы [2] и получена классификационная схема, пригодная для проведения окончательной подклассификации подсемейств уравнения (1). Во-вторых, в качестве примера приложения этой схемы продемонстрировано ее применение к подсемейству квазилинейных уравнений, исследование которых было намечено в работе [4]. При этом получены новые результаты относительно преобразований между уравнениями. Показано, что два уравнения (названные в разделе 4 уравнениями 3В1-2 и 3В1-4), обнаруженные в работе [4], связаны соответственно с уравнениями (3) и (4), а потому оказываются интегрируемыми.

Настоящая работа лежит в русле исследований интегрируемых уравнений с помошью симметрийного подхода, выполняемых многочисленным сообшеством исследователей [2]-[5], [9], [10].

\section{2. ПОЛНАЯ ПРЕДВАРИТЕЛЬНАЯ КЛАССИФИКАЦИЯ}

В этом разделе мы расширяем предварительную классификацию, введенную в работе [2], в которой уравнения (1) переписываются через так называемые нестандартные динамические переменные [11]. Зададим новую динамическую переменную

$$
u=\left(\frac{\partial H}{\partial w_{x x x}}\right)^{-1 / 3}
$$

и введем также дополнительные динамические переменные $v_{1}=w, v_{2}=w_{x}, \ldots, v_{n}=$ $\partial^{n-1} w / \partial x^{n-1}$. Здесь $n=0,1,2$ или 3 в зависимости от того, каков порядок старшей 
производной функции $w$, явно входящей в определение функции $u$. Если $n<3$, то уравнение (1) оказывается квазилинейным. Для квазилинейных уравнений вида

$$
w_{t}=\alpha(x, w) w_{x x x}+\beta\left(x, w, w_{x}, w_{x x}\right)
$$

имеем $n=0$, так что $u=w$, а переменные $v_{i}$ отсутствуют. В случаях $n=1, n=2$ уравнение (1) может быть сведено к соответствуюшему виду:

$$
\begin{aligned}
& w_{t}=\alpha\left(x, w, w_{x}\right) w_{x x x}+\beta\left(x, w, w_{x}, w_{x x}\right), \\
& w_{t}=\alpha\left(x, w, w_{x}, w_{x x}\right) w_{x x x}+\beta\left(x, w, w_{x}, w_{x x}\right) .
\end{aligned}
$$

Для уравнений вида

$$
w_{t}=\alpha(x) w_{x x x}+\beta\left(x, w, w_{x}, w_{x x}\right)
$$

функция $u$ зависит только от $x$ и не задает правильную динамическую переменную. Уравнения типа (10) будут сводиться к уравнениям типа (8) с помошью преобразования $\bar{x}=w, \bar{w}=x$. Наконец, уравнение (1) становится полностью нелинейным в случае, когда $n=3$.

Обозначив $\mathbf{v}=\left(v, v_{1}, \ldots, v_{n}\right)$, можно записать каждое уравнение (1) (в нестандартных переменных $\left.\mathbf{v}, \partial^{i} u / \partial x^{i}\right)$ в виде системы

$$
\mathbf{v}_{x}=(\mathbf{v}, u), \quad \mathbf{v}_{t}=\mathbf{G}\left(\mathbf{v}, u, u_{x}, u_{x x}\right), \quad u_{t}=F\left(\mathbf{v}, u, u_{x}, u_{x x}, u_{x x x}\right) .
$$

Первые условия интегрируемости (5) в нестандартных переменных были найдены в работе [11]:

$$
\begin{gathered}
\rho_{1}=u, \quad \rho_{2}=u^{3} \frac{\partial F}{\partial u_{x x}}, \\
\rho_{3}=\left(2 u^{-2} u_{x}+u^{2} \frac{\partial F}{\partial u_{x x}}\right)_{x}+u^{-3} u_{x}^{2}+\frac{1}{3} u^{5}\left(\frac{\partial F}{\partial u_{x x}}\right)^{2}+u u_{x} \frac{\partial F}{\partial u_{x x}}-u^{2} \frac{\partial F}{\partial u_{x}}+u \sigma_{1}, \\
\rho_{4}=-\frac{1}{3} u_{x x} \frac{\partial F}{\partial u_{x x}}-u_{x} \frac{\partial F}{\partial u_{x}}+u \frac{\partial F}{\partial u}+u^{-1} u_{x}^{2} \frac{\partial F}{\partial u_{x x}}-\frac{1}{3} u^{4} \frac{\partial F}{\partial u_{x}} \frac{\partial F}{\partial u_{x x}}+ \\
+\frac{1}{3} u^{3} u_{x}\left(\frac{\partial F}{\partial u_{x x}}\right)^{2}+\frac{2}{27} u^{7}\left(\frac{\partial F}{\partial u_{x x}}\right)^{3}+\frac{1}{3} u \sigma_{2}, \\
\rho_{5}=u \sigma_{3}-\rho_{3} \sigma_{1}-3 \frac{\partial \Phi}{\partial u}(F) .
\end{gathered}
$$

Вводя обозначение $u_{i}=\partial^{i} u / \partial x^{i}$, можно переписать все уравнения (11), (частично) удовлетворяюшие первым трем условиям, в виде следуюшей системы уравнений [2]:

$$
\begin{aligned}
\mathbf{v}_{x}= & \boldsymbol{\Phi}(\mathbf{v}) \\
\mathbf{v}_{t}= & \left(\frac{u_{2}}{u^{3}}-\frac{3}{2} \frac{u_{1}^{2}}{u^{4}}-\frac{3}{4} P^{-1} \frac{\partial P}{\partial u} \frac{u_{1}^{2}}{u^{3}}-\frac{3}{2} P^{-1} \mathbf{\Phi}(P) \frac{u_{1}}{u^{3}}+E \frac{u_{1}}{u^{3}}\right) \frac{\partial \boldsymbol{\Phi}}{\partial u}- \\
& -\frac{1}{2} \frac{u_{1}^{2}}{u^{3}} \frac{\partial^{2} \mathbf{\Phi}}{\partial u^{2}}-\frac{u_{1}}{u^{3}}\left[\mathbf{\Phi}, \frac{\partial \boldsymbol{\Phi}}{\partial u}\right]+\mathbf{r}(\mathbf{v}, u), \\
u_{t}= & D_{x}\left(\frac{u_{2}}{u^{3}}-\frac{3}{2} \frac{u_{1}^{2}}{u^{4}}-\frac{3}{4} P^{-1} \frac{\partial P}{\partial u} \frac{u_{1}^{2}}{u^{3}}-\frac{3}{2} P^{-1} \mathbf{\Phi}(P) \frac{u_{1}}{u^{3}}+E \frac{u_{1}}{u^{3}}+q(\mathbf{v}, u)\right),
\end{aligned}
$$


условия совместности которой имеют вид

$$
\begin{gathered}
\frac{\partial^{3} \boldsymbol{\Phi}}{\partial u^{3}}+\frac{3}{2} P^{-1} \frac{\partial P}{\partial u} \frac{\partial^{2} \boldsymbol{\Phi}}{\partial u^{2}}=0 \\
{\left[\mathbf{\Phi}, \frac{\partial^{2} \boldsymbol{\Phi}}{\partial u^{2}}\right]+\left(\frac{1}{2} P^{-1} \frac{\partial P}{\partial u}-\frac{1}{u}\right)\left[\boldsymbol{\Phi}, \frac{\partial \boldsymbol{\Phi}}{\partial u}\right]+\left(P^{-1} \frac{\partial P}{\partial u}-\frac{2}{3} E\right) \frac{\partial^{2} \boldsymbol{\Phi}}{\partial u^{2}}=0} \\
\frac{\partial \mathbf{r}}{\partial u}=\frac{\partial q}{\partial u} \frac{\partial \boldsymbol{\Phi}}{\partial u}+\frac{1}{u^{3}}\left[\boldsymbol{\Phi},\left[\boldsymbol{\Phi}, \frac{\partial \boldsymbol{\Phi}}{\partial u}\right]\right]+\frac{3}{2 u^{3}}\left(P^{-1} \frac{\partial P}{\partial u}-\frac{2}{3} E\right)\left[\boldsymbol{\Phi}, \frac{\partial \boldsymbol{\Phi}}{\partial u}\right] \\
{[\boldsymbol{\Phi}, \mathbf{r}]-\mathbf{\Phi}(q) \frac{\partial \boldsymbol{\Phi}}{\partial u}=0 .}
\end{gathered}
$$

При исследовании уравнений совместности, следуя работе [2], можно обнаружить несколько семейств интегрируемых уравнений. В первую очередь, получим три типа уравнений обшего вида $\left(\boldsymbol{\Phi}_{2} \neq 0\right)$

$$
\begin{gathered}
\boldsymbol{\Phi}=u^{2} \boldsymbol{\Phi}_{2}(\mathbf{v})+\boldsymbol{\Phi}_{0}(\mathbf{v}), \quad P=1, \quad E=0, \\
\boldsymbol{\Phi}=S^{1 / 2} \boldsymbol{\Phi}_{2}(\mathbf{v})+\boldsymbol{\Phi}_{0}(\mathbf{v}), \quad S=P^{1 / 2}, \quad P=\alpha(\mathbf{v}) u^{2}-1, \quad E=0, \\
\boldsymbol{\Phi}=u \boldsymbol{\Phi}_{1}(\mathbf{v})+\boldsymbol{\Phi}_{0}(\mathbf{v}), \quad\left[\boldsymbol{\Phi}_{1}, \boldsymbol{\Phi}_{0}\right] \neq 0, \quad P=\alpha(\mathbf{v}) u^{2}, \quad E=E(\mathbf{v}),
\end{gathered}
$$

в которых величина $n$ может принимать значения $0,1,2$ или 3 . Кроме того, можно найти несколько уравнений специального вида (с ограничениями на $n$ ), которые с точностью до локальных преобразований эквивалентности могут быть приведены к следуюшим трем уравнениям:

$$
\begin{aligned}
& \mathbf{\Phi}=\frac{\partial}{\partial x}, \quad u=w, \quad \mathbf{v}=x, \\
& P=\alpha(x) u^{2}+\beta(x) u+\gamma, \quad E=E(x, u), \quad \gamma E=0 \\
& \boldsymbol{\Phi}=\frac{1}{\theta(x) u} \boldsymbol{\Phi}_{2}+u \boldsymbol{\Phi}_{1}+\boldsymbol{\Phi}_{0}, \quad\left[\boldsymbol{\Phi}_{2}, \boldsymbol{\Phi}_{1}\right]=-\frac{2}{3} \varepsilon(x) \boldsymbol{\Phi}_{2}, \\
& {\left[\mathbf{\Phi}_{2}, \boldsymbol{\Phi}_{0}\right]=\frac{1}{\theta} \frac{\partial \theta}{\partial x} \boldsymbol{\Phi}_{2}, \quad P=\theta^{2} u^{2}, \quad E=\varepsilon(x) u ;} \\
& \boldsymbol{\Phi}=\frac{1}{\theta(x) u-1} \boldsymbol{\Phi}_{2}+u \boldsymbol{\Phi}_{1}+\boldsymbol{\Phi}_{0}, \quad\left[\boldsymbol{\Phi}_{2}, \boldsymbol{\Phi}_{0}\right]=\frac{1}{2 \theta}\left[\boldsymbol{\Phi}_{1}, \boldsymbol{\Phi}_{0}\right]+\frac{1}{\theta} \frac{\partial \theta}{\partial x} \boldsymbol{\Phi}_{2}, \\
& {\left[\boldsymbol{\Phi}_{2}, \boldsymbol{\Phi}_{0}\right]=-\frac{1}{2}\left[\boldsymbol{\Phi}_{1}, \boldsymbol{\Phi}_{0}\right], \quad P=(\theta u-1)^{2}, \quad E=0 .}
\end{aligned}
$$

Здесь $\boldsymbol{\Phi}_{2}=a(x, w) \partial / \partial w, \quad \boldsymbol{\Phi}_{1}=b(x, w) \partial / \partial w, \quad \boldsymbol{\Phi}_{0}=\partial / \partial x+c(x, w) \partial / \partial w$ и $n=1$, за исключением уравнения (20), где $n=0$.

Уравнение (11) может быть классифицировано в зависимости от того, принадлежит ли оно к общему типу (тип 1,2 или 3 ) или является уравнением необщего типа. Кроме того, будем называть уравнение принадлежашим классу 0, если это уравнение вида (10), и классу $1,2,3$ или 4 , если $n$ равно соответственно $0,1,2$ или 3 (при этом оно будет или уравнением некоторого уровня квазилинейности, или полностью нелинейным). 
2.1. Эквивалентность и дифференциальные подстановки. Важной концепцией указанной классификации является эквивалентность уравнений. Предполагается, что соотношения эквивалентности суть классические преобразования, т.е. точечные или контактные преобразования $\bar{x}=\phi\left(x, w, w_{x}\right), \quad \bar{w}=\psi\left(x, w, w_{x}\right), \quad$ в которых $D_{x}(\phi) \partial \psi / \partial w_{x}=D_{x}(\psi) \partial \phi / \partial w_{x}$. Такое понятие эквивалентности автоматически продолжается на множество нестандартных переменных, так как переход от стандартных переменных к нестандартным представляет собой обратимое преобразование. Также используются и более общие дифференциальные подстановки

$$
\bar{x}=\phi\left(x, w, w_{x}, w_{x x}, \ldots\right), \quad \bar{w}=\psi\left(x, w, w_{x}, w_{x x}, \ldots\right),
$$

которые могут связывать различные интегрируемые уравнения, хотя и с помошью необратимых преобразований. Само по себе сушествование дифференциальной подстановки, связьваюшей два уравнения, нетривиально, так как такая подстановка может рассматриваться как ограниченньй тип преобразований Беклунда. Например, в п. 3.2 рассматриваются дифференциальные подстановки, сушествуюшие в случае, если уравнение допускает классическую симметрию группы Ли. Исчерпывающая информация о дифференциальных подстановках может быть найдена в работах [7], [12].

2.2. Две схемы классификации. Классы уравнений, описанные выше, не обязательно контактно-инвариантны. Например, полностью нелинейные уравнения образуют класс 4 ( $n=3$ ), который контактно-инвариантен, и, более того, каждое уравнение типа 1, 2 или 3 из класса 4 также контактно-инвариантно. Тем не менее некоторые семейства квазилинейных уравнений могут быть связаны между собой с помошью контактных преобразований. Известно, что всякое уравнение из классов 0, 1 или 2 может быть преобразовано в уравнение из класса 3 . Более того, уравнения класса 3 и типа 2 всегда могут быть преобразованы в уравнения класса 3 и типа 1. Поэтому достаточно распределить уравнения (1) на подсемейства в соответствии с первой схемой, представленной в табл. 1. Эта схема, предложенная в работе [2], хорошо работает в случае, когда имеются мошные вычислительные машины и методы, поскольку вычисление уравнений класса 3 и типа 3 может быть очень трудоемким. Другой подход, описанный в работе [4], состоит в том, чтобы разбить уравнения из этого последнего семейства на несколько подсемейств. Любое уравнение класса 3 и типа 3 с помощью контактного преобразования можно превратить в квазилинейные уравнения класса 0, 1 или 2 вида

$$
w_{t}=\frac{w_{x x x}}{u\left(x, w, w_{x}\right)^{3}}+H\left(x, w, w_{x}, w_{x x}\right),
$$

который инвариантен относительно точечных преобразований $\bar{x}=\phi(x, w), \bar{w}=\psi(x, w)$. Если $\partial^{2} u / \partial w_{1}^{2}=0$, то в зависимости от того, представляет ли собой функция $u$ полную производную или не является таковой, получаются два семейства уравнений:

$$
\begin{aligned}
& w_{t}=w_{x x x}+H\left(x, w, w_{x}, w_{x x}\right), \\
& w_{t}=D_{x}\left(\frac{w_{x x}}{w^{3}}+H\left(x, w, w_{x}\right)\right) .
\end{aligned}
$$

Уравнения (24) добавляют в схему новую ячейку, отвечающую уравнениям класса 0 необщего вида, в то время как уравнения (25) отвечают уравнениям необщего вида уравнения типа (20). Если $\partial^{2} u / \partial w_{1}^{2} \neq 0$, то можно использовать точечные преобразования, 
для того чтобы ограничить поиск новых уравнений на класс 2 и тип 1 или на необщие типы уравнений $(21),(22)$. Получающаяся при этом схема представлена в правой части табл. 1.

ТАБЛИЦА 1

\section{Семейства интегрируемых уравнений}

тип 1 тип 2 тип 3 необшие

класс 0

класс 1

класс 2

класс 3

класс 4

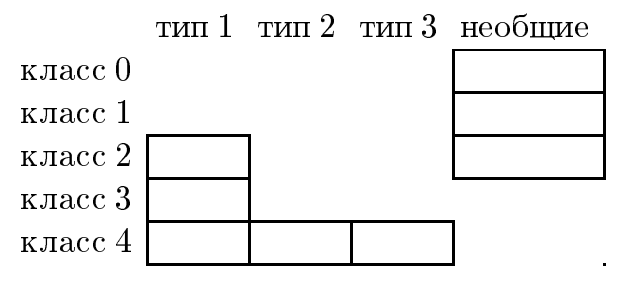

Квазилинейные интегрируемые уравнения были перечислены в работе [4]. В следуюшем разделе будут найдены некоторые явные формулы для уравнений типа 1 и класса 3 и рассмотрение работы [4] будет дополнено анализом преобразований, которые связывают между собой некоторые из этих уравнений. Это подсемейство уравнений появляется в обеих классификационных схемах.

\section{3. УРАВНЕНИЯ ТИПА 1}

Хорошей отправной точкой для построения полной классификации уравнений вида (1) в соответствии с правой схемой в табл. 1 может послужить исследование обшего уравнения типа 1 (уравнение (17)). Из формул (17) и (12) следует, что исследуемые уравнения имеют вид

$$
\begin{aligned}
u_{t} & =D_{x}\left(\frac{u_{2}}{u^{3}}-\frac{3}{2} \frac{u_{1}^{2}}{u^{4}}+q(\mathbf{v}, u)\right), \\
D_{x} \mathbf{v} & =u^{2} \boldsymbol{\Phi}_{2}(\mathbf{v})+\boldsymbol{\Phi}_{0}(\mathbf{v}), \\
\mathbf{v}_{t} & =\left(2 \frac{u_{2}}{u^{2}}-4 \frac{u_{1}^{2}}{u^{3}}\right) \boldsymbol{\Phi}_{2}+2 \frac{u_{1}}{u^{2}}\left[\Phi_{2}, \boldsymbol{\Phi}_{0}\right]+\mathbf{r}(\mathbf{v}, u) .
\end{aligned}
$$

При этом выполняется второе условие интегрируемости, которое принимает вид $\rho_{2}=$ $D_{x}(-6 \ln u)$. Третья каноническая плотность при этом равна

$$
\rho_{3}=D\left(3 \frac{u_{1}}{u^{2}}\right)+\frac{3}{2} \frac{u_{1}^{2}}{u^{3}}+\beta(\mathbf{v}, u), \quad \beta(\mathbf{v}, u)=q u-q_{u} u^{2},
$$

в то время как четвертая каноническая плотность представляет собой $\rho_{4} \sim 2 \boldsymbol{\Phi}_{2}(q) u^{2}$ (где символ $\sim$ означает равенство с точностью до полной $x$-производной). Можно проверить, что

$$
\begin{aligned}
\left(\rho_{3}\right)_{t} \sim & \frac{1}{2}\left(3 q+\beta_{u}\right)_{u u} \frac{u_{1}^{3}}{u^{3}}+\left(\frac{1}{2} \boldsymbol{\Phi}_{2}\left(3 q+\beta_{u}\right) \frac{1}{u^{2}}+\frac{3}{2} \boldsymbol{\Phi}_{2}\left(3 q_{u}+\beta_{u u}\right) \frac{1}{u}-\right. \\
& \left.-\frac{3}{2} \boldsymbol{\Phi}_{0}\left(3 q+\beta_{u}\right) \frac{1}{u^{4}}+\frac{3}{2} \boldsymbol{\Phi}_{0}\left(3 q_{u}+\beta_{u u}\right) \frac{1}{u^{3}}-2 \boldsymbol{\Phi}_{2}\left(\beta_{u}\right) \frac{1}{u^{2}}\right) u_{1}^{2}+\kappa(\mathbf{v}, u) .
\end{aligned}
$$


Коэффиициенты при членах $u_{1}^{3}, u_{1}^{2}$ в формуле (29) вместе с условиями совместности (16) позволяют еще более сузить класс возможных представлений для функций $q(\mathbf{v}, u)$ и $\mathbf{r}(\mathbf{v}, u)$, так что все интегрируемые уравнения типа 1 должны иметь вид

$$
\begin{aligned}
u_{t}= & D_{x}\left(\frac{u_{2}}{u^{3}}-\frac{3}{2} \frac{u_{1}^{2}}{u^{4}}+\frac{Q(\mathbf{v})}{u^{2}}+T(\mathbf{v})+l(\mathbf{v}) u^{2}+m(\mathbf{v}) u\right) \\
\mathbf{v}_{t}= & \left(2 \frac{u_{2}}{u^{2}}-4 \frac{u_{1}^{2}}{u^{3}}\right) \boldsymbol{\Phi}_{2}+2 \frac{u_{1}}{u^{2}}\left[\boldsymbol{\Phi}_{2}, \boldsymbol{\Phi}_{0}\right]+\left(4 \frac{Q(\mathbf{v})}{u}+m(\mathbf{v}) u^{2}+\frac{4}{3} l(\mathbf{v}) u^{3}\right) \boldsymbol{\Phi}_{2}+ \\
& +2\left[\boldsymbol{\Phi}_{0},\left[\boldsymbol{\Phi}_{2}, \boldsymbol{\Phi}_{0}\right]\right] \frac{1}{u}-2\left[\boldsymbol{\Phi}_{2},\left[\boldsymbol{\Phi}_{2}, \boldsymbol{\Phi}_{0}\right]\right] u+\mathbf{s}(\mathbf{v}),
\end{aligned}
$$

где

$$
\begin{aligned}
\boldsymbol{\Phi}_{2}(l) & =0, \\
\boldsymbol{\Phi}_{0}(T)-\boldsymbol{\Phi}_{2}(Q) & =0, \\
\boldsymbol{\Phi}_{2}(m) & =0 .
\end{aligned}
$$

Из формулы (16) следует, что еше имеются условия совместности

$$
\begin{aligned}
& {\left[\boldsymbol{\Phi}_{2},\left[\boldsymbol{\Phi}_{2},\left[\boldsymbol{\Phi}_{2}, \boldsymbol{\Phi}_{0}\right]\right]\right]+\frac{2}{3} l\left[\boldsymbol{\Phi}_{2}, \boldsymbol{\Phi}_{0}\right]+\left(\boldsymbol{\Phi}_{2}(T)+\frac{1}{3} \boldsymbol{\Phi}_{0}(l)\right) \boldsymbol{\Phi}_{2}=0,} \\
& {\left[\boldsymbol{\Phi}_{0},\left[\boldsymbol{\Phi}_{0},\left[\boldsymbol{\Phi}_{2}, \boldsymbol{\Phi}_{0}\right]\right]\right]-2 Q\left[\boldsymbol{\Phi}_{2}, \boldsymbol{\Phi}_{0}\right]+\boldsymbol{\Phi}_{0}(Q) \boldsymbol{\Phi}_{2}=0,} \\
& {\left[\boldsymbol{\Phi}_{2}, \mathbf{s}\right]-m\left[\boldsymbol{\Phi}_{2}, \boldsymbol{\Phi}_{0}\right]-\boldsymbol{\Phi}_{0}(m) \boldsymbol{\Phi}_{2}=0,} \\
& {\left[\boldsymbol{\Phi}_{0}, \mathbf{s}\right]=0 .}
\end{aligned}
$$

Условие интегрируемости (29) все еше не выполнено. Необходимыми и достаточными условиями интегрируемости будут в данном случае условия

$$
\begin{aligned}
0 & =\boldsymbol{\Phi}_{2} \boldsymbol{\Phi}_{2} \boldsymbol{\Phi}_{2}(T)-3 \boldsymbol{\Phi}_{2} \boldsymbol{\Phi}_{2} \boldsymbol{\Phi}_{0}(l)+\frac{8}{3} \boldsymbol{\Phi}_{2}(T) l, \\
0 & =15 \boldsymbol{\Phi}_{0} \boldsymbol{\Phi}_{2} \boldsymbol{\Phi}_{0}(Q)-10 \boldsymbol{\Phi}_{0} \boldsymbol{\Phi}_{0} \boldsymbol{\Phi}_{2}(Q)-6 \boldsymbol{\Phi}_{2} \boldsymbol{\Phi}_{0} \boldsymbol{\Phi}_{0}(Q)+8 \boldsymbol{\Phi}_{2}(Q) Q, \\
\boldsymbol{\Phi}_{2}(E) & =\boldsymbol{\Phi}_{0} \boldsymbol{\Phi}_{2} \boldsymbol{\Phi}_{2}(T)+\boldsymbol{\Phi}_{0} \boldsymbol{\Phi}_{2} \boldsymbol{\Phi}_{0}(l), \\
\boldsymbol{\Phi}_{0}(E) & =2 \boldsymbol{\Phi}_{0} \boldsymbol{\Phi}_{0} \boldsymbol{\Phi}_{2}(T)+\boldsymbol{\Phi}_{2} \boldsymbol{\Phi}_{2} \boldsymbol{\Phi}_{0}(Q)-2 \boldsymbol{\Phi}_{2} \boldsymbol{\Phi}_{0} \boldsymbol{\Phi}_{2}(Q)+\frac{2}{3} \boldsymbol{\Phi}_{0} \boldsymbol{\Phi}_{0} \boldsymbol{\Phi}_{0}(l), \\
\mathbf{s}(l) & =-3 \boldsymbol{\Phi}_{2} \boldsymbol{\Phi}_{2} \boldsymbol{\Phi}_{0}(m)-2 \boldsymbol{\Phi}_{0}(m) l+m \boldsymbol{\Phi}_{0}(l), \\
\mathbf{s}(T) & =3 \boldsymbol{\Phi}_{0} \boldsymbol{\Phi}_{2} \boldsymbol{\Phi}_{0}(m)-3 \boldsymbol{\Phi}_{2} \boldsymbol{\Phi}_{0} \boldsymbol{\Phi}_{0}(m)+m \boldsymbol{\Phi}_{2}(Q), \\
\mathbf{s}(Q) & =-\boldsymbol{\Phi}_{0} \boldsymbol{\Phi}_{0} \boldsymbol{\Phi}_{0}(m)+\boldsymbol{\Phi}_{0}(Q) m+2 Q \boldsymbol{\Phi}_{0}(m),
\end{aligned}
$$

где $E=E(\mathbf{v}, u)$ представляет собой функцию

$$
E(\mathbf{v}, u)=\frac{5}{3} \boldsymbol{\Phi}_{0} \boldsymbol{\Phi}_{0}(l)+\frac{7}{3} \boldsymbol{\Phi}_{0} \boldsymbol{\Phi}_{2}(T)+\frac{4}{3} \boldsymbol{\Phi}_{2} \boldsymbol{\Phi}_{0}(T)+\frac{1}{6} T^{2}-l Q .
$$

Вышеприведенные условия интегрируемости достаточны, чтобы можно было применить нашу классификацию. Для некоторого простого уравнения (класса 0) условие, связанное с величиной $\rho_{5}$, оказывается необходимым. 
3.1. Классические симметрии. "Хвосты" уравнений (30), (31), т.е. члены, зависяшие от $m(\mathbf{v})$ и $\mathbf{s}(\mathbf{v})$, суть классические симметрии уравнений $(30),(31)$. Эти симметрии могут быть представлены в виде

$$
u_{t}=D_{x}(m(\mathbf{v}) u), \quad v_{t}=m(\mathbf{v}) u^{2} \boldsymbol{\Phi}_{2}+\mathbf{s}(\mathbf{v}) .
$$

Тем самым, если $m(\mathbf{v})$ и $\mathbf{s}(\mathbf{v})$ удовлетворяют условиям совместности $(37),(38)$ и условию (34), то (46) представляет собой классическую симметрию, и наоборот. Условия (43)-(45) позволяют найти классические симметрии интегрируемых уравнений.

3.2. Дифференциальные подстановки для уравнения КдФ. Покажем теперь, как найти дифференциальные подстановки, которые переводят уравнения (30), (31) в уравнение, тесно связанное с уравнением $\mathrm{K} д \Phi$ (3). Если уравнение эволюции обладает классической симметрией, то сушествует дифференшиальная подстановка (23), которая приводит это уравнение к уравнению с ме́ншшей группой симметрий. Это так называемые групповые преобразования [7]. Проблема, связанная с исходным уравнением КдФ (3), состоит в том, что это уравнение имеет классические симметрии, а потому должно существовать "более фундаментальное" уравнение, связанное с уравнением Кд $\Phi$ с помощью групповой подстановки и имеющее тривиальную группу симметрий. Это уравнение имеет вид

$$
w_{t}=D_{y}\left(\frac{w_{y y}}{w^{3}}-3 \frac{w_{y}^{2}}{w^{4}}-3 y\right),
$$

и оно получается из уравнения КдФ при подстановке $w=1 / u_{x}, y=u$ и масштабном преобразовании.

Общее свойство дифференциальных подстановок (23) заключается в том, что если начальное уравнение (в данном случае уравнение (30)) имеет сохраняюшуюся плотность $\rho$ (следовательно, $\rho_{t}=\sigma_{x}$ ), то преобразованное уравнение имеет сохраняюшуюся плотность $\bar{\rho}=\rho / D_{x} \phi$. Теперь $u$ есть сохраняюшаяся плотность уравнения (30), а потому она должна преобразовываться в сохраняюшуюся плотность $w$ уравнения (47), поэтому подстановка должна иметь вид

$$
y=Z, \quad w=\frac{u}{D_{x} Z}
$$

Налагая условие, что дифференшиальная подстановка (23) должна переводить (30) в (47), получим, что

$$
Z_{t}=\frac{D_{x} Z}{u}\left(\frac{Q}{u^{2}}+T+l u^{2}+m u\right)+\frac{D_{x}^{3} Z}{u^{3}}-3 D_{x}^{2} Z \frac{u_{1}}{u^{4}}+\frac{3}{2} D_{x} Z \frac{u_{1}^{2}}{u^{5}}+3 \frac{Z D_{x} Z}{u}
$$

и возможны два типа подстановок. Первьй тип, обозначаемьй как КдФ1, имеет вид

$$
Z=\frac{1}{u} D_{x}\left(-\frac{u_{1}}{u^{2}}+N(\mathbf{v}) u\right)-\frac{1}{2} \frac{u_{1}^{2}}{u^{4}}-\frac{Q}{u^{2}}-\frac{1}{3} T+\frac{1}{3} l u^{2},
$$


где $N=N(\mathbf{v})$. Из условий совместности вытекают следуюшие необходимые и достаточные условия:

$$
\begin{gathered}
\boldsymbol{\Phi}_{2}(N)=-\frac{2}{3} l-\frac{1}{4} N^{2}, \\
0=6 \boldsymbol{\Phi}_{2} \boldsymbol{\Phi}_{2} \boldsymbol{\Phi}_{0}(N)+3 N \boldsymbol{\Phi}_{2} \boldsymbol{\Phi}_{0}(N)-2 \boldsymbol{\Phi}_{2}(T) N+2 \boldsymbol{\Phi}_{2} \boldsymbol{\Phi}_{2}(T)+6 \boldsymbol{\Phi}_{2} \boldsymbol{\Phi}_{0}(l), \\
0=3 \boldsymbol{\Phi}_{0} \boldsymbol{\Phi}_{2} \boldsymbol{\Phi}_{0}(N)-3 \boldsymbol{\Phi}_{2} \boldsymbol{\Phi}_{0} \boldsymbol{\Phi}_{0}(N)+\frac{3}{2} N \boldsymbol{\Phi}_{0} \boldsymbol{\Phi}_{0}(N)+2 \boldsymbol{\Phi}_{2}(Q) N- \\
-\frac{3}{2} Q N^{2}-2 \boldsymbol{\Phi}_{0} \boldsymbol{\Phi}_{2}(T)-\frac{4}{3} Q l+E, \\
0=\boldsymbol{\Phi}_{0} \boldsymbol{\Phi}_{0} \boldsymbol{\Phi}_{0}(N)-2 Q \boldsymbol{\Phi}_{0}(N)-\boldsymbol{\Phi}_{0}(Q) N+2 \boldsymbol{\Phi}_{0} \boldsymbol{\Phi}_{2}(Q)-\boldsymbol{\Phi}_{2} \boldsymbol{\Phi}_{0}(Q), \\
\mathbf{s}(N)=\boldsymbol{\Phi}_{0}(N) m-\boldsymbol{\Phi}_{0}(m) N+2 \boldsymbol{\Phi}_{2} \boldsymbol{\Phi}_{0}(m) .
\end{gathered}
$$

Для подстановок второго типа, обозначаемых как КдФ2, получим

$$
Z=\frac{1}{u} D_{x}\left(\frac{u_{1}}{u^{2}}+\frac{N(\mathbf{v})}{u}\right)-\frac{1}{2} \frac{u_{1}^{2}}{u^{4}}-\frac{Q}{u^{2}}-\frac{1}{3} T+\frac{1}{3} l u^{2}
$$

где $N=N(\mathbf{v})$, и

$$
\begin{gathered}
\boldsymbol{\Phi}_{0}(N)=2 Q-\frac{1}{4} N^{2}, \\
0=\boldsymbol{\Phi}_{2} \boldsymbol{\Phi}_{2} \boldsymbol{\Phi}_{2}(N)+\frac{2}{3} l \boldsymbol{\Phi}_{2}(N)-\boldsymbol{\Phi}_{2} \boldsymbol{\Phi}_{2}(T)-\boldsymbol{\Phi}_{2} \boldsymbol{\Phi}_{0}(l), \\
0=3 \boldsymbol{\Phi}_{2} \boldsymbol{\Phi}_{0} \boldsymbol{\Phi}_{2}(N)-3 \boldsymbol{\Phi}_{0} \boldsymbol{\Phi}_{2} \boldsymbol{\Phi}_{2}(N)+\frac{3}{2} N \boldsymbol{\Phi}_{2} \boldsymbol{\Phi}_{2}(N)-N \boldsymbol{\Phi}_{2}(T)- \\
-N \boldsymbol{\Phi}_{0}(l)+\frac{1}{2} l N^{2}-2 \boldsymbol{\Phi}_{2} \boldsymbol{\Phi}_{2}(Q)-\frac{4}{3} Q l+E, \\
0=3 \boldsymbol{\Phi}_{0} \boldsymbol{\Phi}_{0} \boldsymbol{\Phi}_{2}(N)+\frac{3}{2} N \boldsymbol{\Phi}_{0} \boldsymbol{\Phi}_{2}(N)-N \boldsymbol{\Phi}_{2}(Q)+3 \boldsymbol{\Phi}_{2} \boldsymbol{\Phi}_{0}(Q)-8 \boldsymbol{\Phi}_{0} \boldsymbol{\Phi}_{2}(Q), \\
\quad \mathbf{s}(N)=-\frac{1}{4} N^{2} m+N \boldsymbol{\Phi}_{0}(m)+2 Q m-2 \boldsymbol{\Phi}_{0} \boldsymbol{\Phi}_{0}(m) .
\end{gathered}
$$

3.3. Дифференциальные подстановки для уравнения КН. Несимметричная версия уравнения $\mathrm{KH}$ (4) имеет вид

$$
w_{t}=D_{y}\left(\frac{w_{y y}}{w^{3}}-\frac{3}{2} \frac{w_{y}^{2}}{w^{4}}+R(y) w^{2}\right), \quad R(y)=y^{3}+\lambda_{1} y+\lambda_{0}
$$

Дифференциальная подстановка при этом также имеет вид (48), в котором

$$
Z_{t}=\frac{D_{x} Z}{u}\left(\frac{Q}{u^{2}}+T+l u^{2}+m u\right)+\frac{1}{u^{3}}\left(D_{x}^{3} Z-\frac{3}{2} \frac{\left(D_{x}^{2} Z\right)^{2}}{D_{x} Z}\right)-\frac{R(Z)}{D_{x} Z} u
$$

4 Теоретическая и математическая физика, т. 133, № 2, 2002 г. 
При этом $Z=Z(\mathbf{v})$, и опять возможны два типа дифференциальных подстановок. Для первого типа подстановок, обозначаемого как КН1, характерно сушествование непостоянной функции $Z(\mathbf{v})$, удовлетворяющей системе уравнений

$$
\begin{gathered}
\boldsymbol{\Phi}_{2}(Z)=0 \\
0=\boldsymbol{\Phi}_{2} \boldsymbol{\Phi}_{2} \boldsymbol{\Phi}_{0}(Z)-\frac{1}{2} \frac{\left(\boldsymbol{\Phi}_{2} \boldsymbol{\Phi}_{0}(Z)\right)^{2}}{\boldsymbol{\Phi}_{0}(Z)}+\frac{1}{3} l \boldsymbol{\Phi}_{0}(Z)-\frac{1}{3} \frac{R(Z)}{\boldsymbol{\Phi}_{0}(Z)} \\
0=\boldsymbol{\Phi}_{2} \boldsymbol{\Phi}_{0} \boldsymbol{\Phi}_{0}(Z)-\boldsymbol{\Phi}_{0} \boldsymbol{\Phi}_{2} \boldsymbol{\Phi}_{0}(Z)-\frac{\boldsymbol{\Phi}_{2} \boldsymbol{\Phi}_{0}(Z) \boldsymbol{\Phi}_{0} \boldsymbol{\Phi}_{0}(Z)}{\boldsymbol{\Phi}_{0}(Z)}+\frac{1}{3} T \boldsymbol{\Phi}_{0}(Z), \\
0=\boldsymbol{\Phi}_{0} \boldsymbol{\Phi}_{0} \boldsymbol{\Phi}_{0}(Z)-\frac{3}{2} \frac{\left(\boldsymbol{\Phi}_{0} \boldsymbol{\Phi}_{0}(Z)\right)^{2}}{\boldsymbol{\Phi}_{0}(Z)}+Q \boldsymbol{\Phi}_{0}(Z), \\
\mathbf{s}(Z)=m \boldsymbol{\Phi}_{0}(Z) .
\end{gathered}
$$

В случае подстановки типа КН2 функция $Z(\mathbf{v})$ должна удовлетворять системе

$$
\begin{gathered}
\boldsymbol{\Phi}_{0}(Z)=0 \\
0=\boldsymbol{\Phi}_{0} \boldsymbol{\Phi}_{0} \boldsymbol{\Phi}_{2}(Z)-\frac{1}{2} \frac{\left(\boldsymbol{\Phi}_{0} \boldsymbol{\Phi}_{2}(Z)\right)^{2}}{\boldsymbol{\Phi}_{2}(Z)}-Q \boldsymbol{\Phi}_{2}(Z)-\frac{1}{3} \frac{R(Z)}{\boldsymbol{\Phi}_{2}(Z)} \\
0=\boldsymbol{\Phi}_{0} \boldsymbol{\Phi}_{2} \boldsymbol{\Phi}_{2}(Z)-\boldsymbol{\Phi}_{2} \boldsymbol{\Phi}_{0} \boldsymbol{\Phi}_{2}(Z)-\frac{\boldsymbol{\Phi}_{2} \boldsymbol{\Phi}_{2}(Z) \boldsymbol{\Phi}_{0} \boldsymbol{\Phi}_{2}(Z)}{\boldsymbol{\Phi}_{2}(Z)}+\frac{1}{3} T \boldsymbol{\Phi}_{2}(Z), \\
0=\boldsymbol{\Phi}_{2} \boldsymbol{\Phi}_{2} \boldsymbol{\Phi}_{2}(Z)-\frac{3}{2} \frac{\left(\boldsymbol{\Phi}_{2} \boldsymbol{\Phi}_{2}(Z)\right)^{2}}{\boldsymbol{\Phi}_{2}(Z)}-\frac{1}{3} l \boldsymbol{\Phi}_{2}(Z), \\
\mathbf{s}(Z)=0 .
\end{gathered}
$$

\section{4. КВАЗИЛИНЕЙНЫЕ УРАВНЕНИЯ}

Дадим теперь набросок схемы классификации уравнений типа 1 (17) и квазилинейных уравнений

$$
w_{t}=A(\mathbf{v}, u) w_{3}+B(\mathbf{v}, u), \quad w_{i} \equiv \frac{\partial^{i} w}{\partial x^{i}},
$$

но ограничим реальный поиск случаем двух уравнений, про которые ранее не было известно, что они относятся к классу интегрируемых. В нестандартных переменных это семейство уравнений может быть записано в виде

$$
\begin{aligned}
u= & A(\mathbf{v})^{-1 / 3} \\
u_{t}= & D_{x}\left(\frac{u_{2}}{u^{3}}-\frac{3}{2} \frac{u_{1}^{2}}{u^{4}}+\frac{Q(\mathbf{v})}{u^{2}}+T(\mathbf{v})+l(\mathbf{v}) u^{2}+m(\mathbf{v}) u\right), \\
D_{x} \mathbf{v}= & \boldsymbol{\Phi}(\mathbf{v})=u^{2} \boldsymbol{\Phi}_{2}(\mathbf{v})+\boldsymbol{\Phi}_{0}(\mathbf{v}), \\
\mathbf{v}_{t}= & \left(2 \frac{u_{2}}{u^{2}}-4 \frac{u_{1}^{2}}{u^{3}}+4 \frac{Q(\mathbf{v})}{u}+m(\mathbf{v}) u^{2}+\frac{4}{3} l(\mathbf{v}) u^{3}\right) \mathbf{\Phi}_{2}+2 \frac{u_{1}}{u^{2}}\left[\boldsymbol{\Phi}_{2}, \boldsymbol{\Phi}_{0}\right]+ \\
& +2\left[\mathbf{\Phi}_{0},\left[\boldsymbol{\Phi}_{2}, \mathbf{\Phi}_{0}\right]\right] \frac{1}{u}-2\left[\boldsymbol{\Phi}_{2},\left[\boldsymbol{\Phi}_{2}, \boldsymbol{\Phi}_{0}\right]\right] u+\mathbf{s}(\mathbf{v}) .
\end{aligned}
$$


Уравнения класса 3 типа 1 задаются системой

$$
\begin{gathered}
w_{t}=A\left(x, w, w_{1}, w_{2}\right) w_{3}+B\left(x, w, w_{1}, w_{2}\right), \\
\boldsymbol{\Phi}_{2}=a\left(x, w, w_{1}\right) \partial_{w_{1}}, \quad \mathbf{\Phi}_{0}=\partial_{x}+w_{1} \partial_{w}-b\left(x, w, w_{1}\right) \partial_{w_{1}} \\
A\left(x, w, w_{1}, w_{2}\right)=u^{-3}=\left(\frac{w_{2}+b\left(x, w, w_{1}, w_{2}\right)}{a\left(x, w, w_{1}, w_{2}\right)}\right)^{-3 / 2} .
\end{gathered}
$$

Точечные преобразования, сохраняющие вид этих уравнений, суть

$$
\begin{gathered}
\bar{t}=k t, \quad \bar{x}=\phi(x, w), \quad \bar{w}=\psi(x, w), \\
J \equiv \phi_{x} \psi_{w}-\phi_{w} \psi_{x} \neq 0 .
\end{gathered}
$$

Также ниже используются некоторые контактные преобразования, имеющие вид (49).

Первая часть классификации состоит в разрешении условий интегрируемости (32), (33), (39)-(42) и условий (35) и (36). Во-первых, из уравнения (35) следует, что $l=$ $-3\left(a^{2}\right)_{w_{1} w_{1}} / 4$, и тогда уравнение (32) дает $\left(a^{2}\right) w_{1} w_{1} w_{1}$, т.е. $a=\left(\alpha_{2} w_{1}^{2}+\alpha_{1} w_{1}+\alpha_{0}\right)^{1 / 2}$, $\alpha_{i}=\alpha_{i}(x, w)$. Два различных случая соответствуют тому, имеет ли многочлен по $w_{1}$ два простых корня или один двойной. Преобразования (49) позволяют записать эти два случая в соответствуюших формах $a=1$ или $a=\alpha(x, w)^{-1 / 2} w_{1}^{1 / 2}$, которые будут называться соответственно классами 3 А или $3 \mathrm{~B}$. В обоих случаях $l=0$.

Нас интересуют уравнения класса 3 В. Для этого класса получим $a=$ $\alpha(x, w)^{-1 / 2} w_{1}^{1 / 2}$. Тогда из условия (39) следует, что

$$
T=t_{2}(x, w) w_{1}+t_{1}(x, w) w_{1}^{1 / 2}+t_{0}(x, w),
$$

а из условия (35)-

$$
\begin{gathered}
b=\beta_{5} w^{5 / 2}+\beta_{4} w^{2}+\beta_{3} w^{3 / 2}+\beta_{2} w+\beta_{1} w^{1 / 2}, \quad \beta_{i}=\beta_{i}(x, w), \\
t_{2}=3 \beta_{5} \alpha^{-1 / 2}, \quad t_{1}=\frac{3}{2} \beta_{4} \alpha^{-1 / 2}-\frac{3}{2} \alpha_{w} \alpha^{-3 / 2} .
\end{gathered}
$$

Условие (36) позволяет найти явный вид функции $Q$, и из условия (33) следует система

$$
\begin{gathered}
\left(\beta_{5} \alpha^{-3 / 2}\right)_{w}=0, \quad\left(\beta_{1} \alpha^{-3 / 2}\right)_{x}=0, \quad\left(\beta_{2}\right)_{w}+\left(\beta_{4}\right)_{x}=0 \\
\left(t_{0}\right)_{w}+\frac{3 \alpha^{-3 / 2}}{16}\left[\beta_{5} \alpha_{x}+2\left(\beta_{5}\right)_{x} \alpha-2\left(\beta_{3}\right)_{w} \alpha+\beta_{3} \alpha_{w}\right]=0 \\
\left(t_{0}\right)_{x}+\frac{3 \alpha^{-3 / 2}}{16}\left[\beta_{1} \alpha_{w}+2\left(\beta_{1}\right)_{w} \alpha-2\left(\beta_{3}\right)_{x} \alpha+\beta_{3} \alpha_{x}\right]=0 .
\end{gathered}
$$

Разрешая уравнения (50) и используя допустимые преобразования, положим

$$
\begin{array}{lll}
\beta_{5}=\lambda_{1} \alpha^{3 / 2}, & \beta_{1}=\lambda_{2} \alpha^{3 / 2}, & \lambda_{1}, \lambda_{2} \in\{0,1\}, \\
\beta_{4}=\beta_{w}+\frac{\alpha_{w}}{\alpha}, & \beta_{2}=-\beta_{x}-\frac{\alpha_{x}}{\alpha}, & \beta_{3}=\gamma \alpha^{1 / 2},
\end{array}
$$

где $\beta(x, w)$ и $\gamma(x, w)$ - произвольные функции. Уравнения $(51),(52)$ при этом принимают вид

$$
\left(t_{0}\right)_{w}=\frac{3}{8} \gamma_{w}-\frac{3}{4} \lambda_{1} \alpha_{x}, \quad\left(t_{0}\right)_{x}=\frac{3}{8} \gamma_{x}-\frac{3}{4} \lambda_{2} \alpha_{w}
$$


Тогда уравнения класса 3В задаются системой уравнений

$$
\begin{aligned}
& a=\alpha^{-1 / 2} w_{1}^{1 / 2} \\
& b=\lambda_{1} \alpha^{3 / 2} w_{1}^{5 / 2}+\left(\beta_{w}+\frac{\alpha_{w}}{\alpha}\right) w_{1}^{2}+\gamma \alpha^{1 / 2} w_{1}^{-3 / 2}-\left(\beta_{x}+\frac{\alpha_{x}}{\alpha}\right) w_{1}+\lambda_{2} \alpha^{3 / 2} w_{1}^{1 / 2}
\end{aligned}
$$

и могут быть классифицированы, если определить три функции $\alpha(x, w), \beta(x, w)$ и $\gamma(x, w)$ и две константы $\lambda_{1}$ и $\lambda_{2}$. Получим три основных случая:

$$
\begin{aligned}
& \left.\lambda_{1}=1, \quad \lambda_{2}=1 \quad \text { (уравнения } 3 \mathrm{~B} 1\right), \\
& \left.\lambda_{1}=0, \quad \lambda_{2}=1 \quad \text { (уравнения } 3 \mathrm{~B} 2\right), \\
& \left.\lambda_{1}=0, \quad \lambda_{2}=0 \quad \text { (уравнения } 3 \mathrm{~B} 3\right) \text {. }
\end{aligned}
$$

Есть также дополнительный случай, когда уравнения вырождаются, который будем на-

\begin{tabular}{|c|c|}
\hline & Функции $\alpha, \beta, \gamma$ \\
\hline Уравнение 3В1-1 & $\alpha=\alpha(w+x), \quad \beta=\beta(w+x), \quad \gamma=\gamma(w+x)$ \\
\hline Уравнение 3В1-2 & $\begin{array}{l}\alpha=f(w+x)-g(w-x), \quad \beta=0, \quad \gamma=10\left(f^{2}-g^{2}\right), \\
\text { где } \\
\qquad\left(f^{\prime}\right)^{2}+16 f^{5}+2 \lambda f^{3}+\lambda_{2} f^{2}+\lambda_{1} f+\lambda_{0}=0 \\
\quad\left(g^{\prime}\right)^{2}+16 g^{5}+2 \lambda g^{3}+\lambda_{2} g^{2}+\lambda_{1} g+\lambda_{0}=0\end{array}$ \\
\hline Уравнение 3В1-3 & $\begin{array}{l}\alpha=\alpha(w+x), \quad \beta=-\ln \alpha(w+x)-\ln \delta(w-x), \\
\gamma=2 \alpha^{2}(w+x)\end{array}$ \\
\hline Уравнение 3В1-4 & $\begin{array}{l}\alpha=f(w+x)-g(w-x) \\
\beta=-\ln f(w+x)-\ln g(w-x) \\
\gamma=2(f-g)(f+g), \\
\text { где } \\
\quad\left(f^{\prime}\right)^{2}+4 f^{5}+2 \lambda_{4} f^{4}+\lambda_{3} f^{3}+\lambda_{2} f^{2}+\lambda_{1} f=0, \\
\quad\left(g^{\prime}\right)^{2}+4 g^{5}+2 \lambda_{4} g^{4}+\lambda_{3} g^{3}+\lambda_{2} g^{2}+\lambda_{1} g=0\end{array}$ \\
\hline
\end{tabular}
зывать случаем 3В0. В этом случае $a$ и $b$ зависят только от $w_{1}$, так что $\alpha$ и $\gamma$ суть константы, а $\beta$ оказывается линейной функцией от $w_{1}$ и $x$.

ТАБЛИЦА 2

\section{Интегрируемые уравнения класса 3B1}

Уравнения, которые нас интересуют, принадлежат классу 3В1. Этот класс содержит уравнения, приведенные в табл. 2 . Функции $\alpha(w+x), \beta(w+x)$ и $\gamma(w+x)$ из уравнения 3В1-1 должны удовлетворять следуюшей системе:

$$
\begin{aligned}
& \gamma=-\frac{1}{\alpha}\left(2 \beta^{\prime \prime}-\left(\beta^{\prime}\right)^{2}+2 \frac{\alpha^{\prime}}{\alpha} \beta^{\prime}\right)+10 \alpha^{2}+\lambda \alpha \\
& \gamma^{\prime \prime}-\left(\frac{1}{2} \beta^{\prime}+3 \frac{\alpha^{\prime}}{\alpha}\right) \gamma^{\prime}+\left(-2 \beta^{\prime}+\frac{5}{2} \frac{\alpha^{\prime}}{\alpha} \beta^{\prime}-\frac{\alpha^{\prime \prime}}{\alpha}+3 \frac{\left(\alpha^{\prime}\right)^{2}}{\alpha^{2}}\right) \gamma-
\end{aligned}
$$




$$
\begin{gathered}
-4 \alpha^{2} \beta^{\prime \prime}-3 \alpha \alpha^{\prime} \beta^{\prime}-10 \alpha \alpha^{\prime \prime}+10\left(\alpha^{\prime}\right)^{2}=0 \\
\left(\frac{\beta^{\prime \prime}}{\alpha}-3 \frac{\left(\beta^{\prime}\right)^{2}}{\alpha}+4 \frac{\alpha^{\prime \prime}}{\alpha^{2}}-4 \frac{\left(\alpha^{\prime}\right)^{2}}{\alpha^{3}}+\frac{3}{4} \frac{\gamma^{2}}{\alpha^{2}}-\frac{3}{2} \alpha^{2}\right)^{\prime}+ \\
+5 \frac{\beta^{\prime}}{\alpha}\left(\beta^{\prime \prime}+\alpha^{\prime \prime} \alpha-\frac{\left(\alpha^{\prime}\right)^{2}}{\alpha^{2}}\right)+\frac{7}{2} \gamma^{\prime}-\frac{5}{2} \frac{\alpha^{\prime}}{\alpha} \gamma=0
\end{gathered}
$$

Для уравнения 3В1-3 получим

$$
\left(\delta^{\prime}\right)^{2}+\lambda_{2} \delta^{2}+\lambda_{1} \delta=0, \quad\left(\alpha^{\prime}\right)^{2}+4 \alpha^{5}+\lambda_{4} \alpha^{4}+\lambda_{3} \alpha^{3}+\lambda_{2} \alpha^{2}=0 .
$$

\begin{tabular}{|c|c|}
\hline & Дифференциальные подстановки \\
\hline Уравнение 3В1-2 & 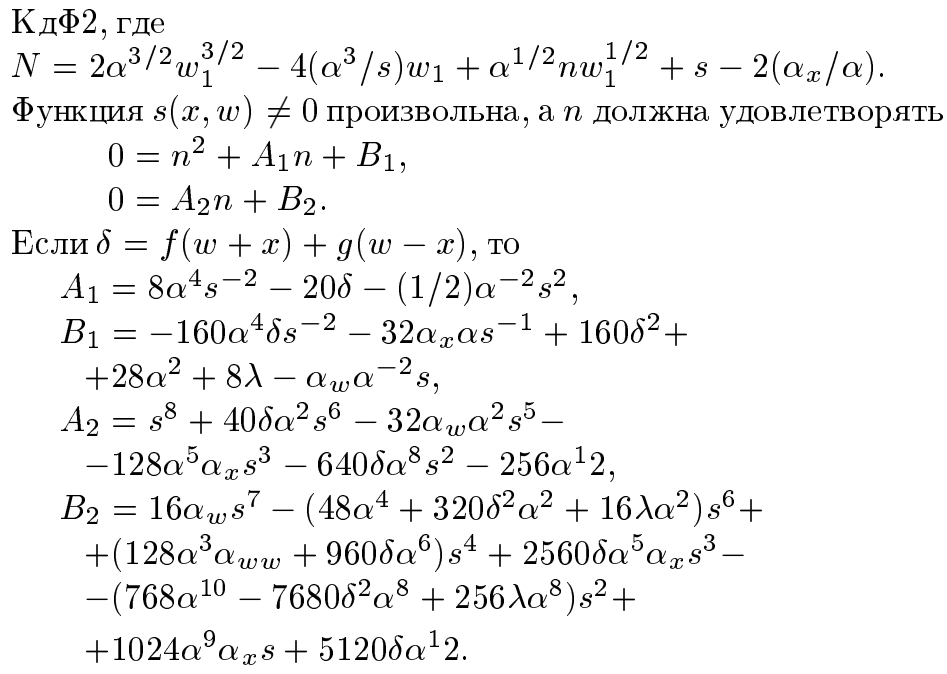 \\
\hline Уравнение 3В1-3 & $\begin{array}{l}\text { КН1, где } Z=\alpha(w+x) \text { и многочлен } R(x) \text { есть } \\
R(x)=-(3 / 2)\left(4 x^{4}+\lambda_{4} x^{3}+\lambda_{3} x^{2}+\lambda_{2} x\right)\end{array}$ \\
\hline Уравнение 3В1-4 & $\begin{array}{l}\text { КН1, где } Z=f(w+x)+g(w-x) \text { и многочлен } R(x) \text { есть } \\
R(x)=-(3 / 2)\left(4 x^{4}+\lambda_{4} x^{3}+\lambda_{3} x^{2}+\lambda_{2} x\right)\end{array}$ \\
\hline Уравнение 3В3-4 & Кд $\Phi 1$, где $N=0$ \\
\hline
\end{tabular}

ТАБЛИЦА 3

Дифференциальные подстановки для уравнений класса 3В1 без классических симметрий

Симметрии и дифференциальные подстановки класса 3В. Из условий симметрии следует, что $m=m(x), \mathbf{s}=s \partial_{w}+\left(s_{x}-b s_{w_{1}}\right) \partial_{w_{1}}$, где $s\left(x, w, w_{1}\right)=m(x) w_{1}+$ 
$n(x)$, и должна выполняться система уравнений

$$
\begin{aligned}
& 0=m^{\prime \prime} w_{1}+n^{\prime \prime}-2 b m^{\prime}+n b_{w} b_{w_{1}}\left(m^{\prime} w_{1}+n^{\prime}\right) \\
& 0=n T_{w}+\left(m^{\prime} w_{1}+n^{\prime}\right) T_{w_{1}} \\
& 0=m^{\prime \prime \prime}+n Q_{w}+\left(m^{\prime} w_{1}+n^{\prime}\right) Q_{w_{1}}-2 m^{\prime} Q
\end{aligned}
$$

Решая эту систему, получим, что из всего класса 3В1 одно лишь уравнение 3В1-1 допускает симметрию, причем вполне очевидную, $m=n=k$.

Подводя итог, в табл. 3 мы собрали дифференциальные подстановки для уравнений семейства 3В1, которые связывают уравнения из этого семейства с уравнениями КдФ и КН. Приведены только те подстановки, которые соответствуют уравнениям без классических симмметрий. Это сделано, во-первых, для краткости, а во-вторых, из-за того, что наилучшим способом обработки уравнений, обладающих классическими симметриями, служит нахождение групповых преобразований (см. [7], [13]). Заметим, что было ранее неизвестно, что уравнения 3В1-2 и 3В1-4 связаны с соответствуюшими уравнениями КдФ и КН, равно как и то, являются ли вообще эти уравнения интегрируемыми. В работе [4] было только показано, что они удовлетворяют некоторым первым условиям интегрируемости, но полная интегрируемость оставалась под вопросом до тех пор, пока не была найдена связь между этими уравнениями и интегрируемыми уравнениями с помощью дифференциальной подстановки.

Благодарности. Автор глубоко признателен С. И. Свинолупову и В.В. Соколову за сотрудничество. Работа была поддержана DGCYT (проект РB98-0821) и Институтом математических наук Исаака Ньютона (Кембридж, Великобритания).

\section{Список литературы}

[1] L. Abellanas, A. Galindo. Phys. Lett. A. 1985. V. 107. № 4. P. 159-160; F. Calogero, A. Degasperis. J. Math. Phys. 1981. V. 22. P. 23-31; A.S. Fokas. J. Math. Phys. 1980. V. 21. № 6. P. 1318-1325.

[2] R. Hernández Heredero, V. V. Sokolov, S. I. Svinolupov. J. Phys. A. 1994. V. 27. P. 4557-4568.

[3] Н. Х. Ибрагимов, А. Б. Шабат. Функц. анализ и его прилож. 1980. Т. 14. № 1. С. 25-36.

[4] A.V. Mikhailov, A.B. Shabat, V. V. Sokolov. The symmetry approach to classification of integrable equations. In: What is Integrability? Ed. V. Zakharov. Berlin: Springer, 1991. P. 115-184.

[5] С. И. Свинолупов, В. В. Соколов. Функц. анализ и его прилож. 1982. Т. 16. № 4. С. 86-87.

[6] H.D. Wahlquist, F. B. Estabrook. J. Math. Phys. 1975. V. 16. P. 1-7.

[7] С.И. Свинолупов, В. В. Соколов. УМН. 1992. Т. 47. № 3. С. 115-146.

[8] H. H. Chen, Y. C. Lee, C.S. Liu. Physica Scripta. 1979. V. 20. № 3/4. P. 490-492.

[9] Н. Х. Ибрагимов, А. Б. Шабат. Функц. анализ и его прилож. 1980. Т. 14. № 4. С. 79-80.

[10] V. V. Sokolov, A. B. Shabat. Sov. Math. Phys. Rev. 1984. V. 4. P. 221-280.

[11] Ф. Х. Мукминов, В. В. Соколов. Матем. сб. 1987. Т. 133(175). № 3. С. 392-414.

[12] В. В. Соколов. УМН. 1988. Т. 43. № 5. С. 133-163.

[13] R. Hernández Heredero, V. V. Sokolov, S. I. Svinolupov. Why are there so many integrable evolution equations of third order? In: Proc. NEEDS'94. Eds. V.G. Manakov, A.R. Bishop, D.D. Holm. Los Álamos: World Scientific, 1995. P. 42-53. 